\title{
ANTHROPEN
}

Le dictionnaire francophone d'anthropologie ancré dans le contemporain

\section{RECHERCHE PARTICIPATIVE}

\author{
Lamoureux, Ève
}

UQAM, Canada

Date de publication : 2021-02-02

DOI : https://doi.org/10.47854/anthropen.vi0.51166

Voir d'autres entrées dans le dictionnaire

La recherche participative s'emploie à analyser des phénomènes concrets et complexes issus du terrain et à le faire en mobilisant des acteurs et actrices, des savoirs et des expertises diversifiés. Elle regroupe des pratiques aux fondements épistémologiques multiples et aux approches méthodologiques variées qui prennent plusieurs appellations - recherche-action, collaborative, évaluative, ancrée dans la communauté, etc. - ce qui en fait un concept polysémique.

Inspirée de la philosophie pragmatique de Dewey (1927), la recherche participative consiste à développer une connaissance au service de la pratique, c'està-dire d'abord orientée vers une logique d'action. Cette connaissance s'ancre, d'une part, dans une perspective rationnelle technique: combiner les expertises des personnes concernées afin d'engendrer de nouveaux savoirs et pratiques efficaces (Anadón 2006). Un pendant plus critique, d'autre part, associe la recherche participative aux luttes contre les divers systèmes de domination - classisme, (hétéro)sexisme, racisme, capacitisme, etc. - en promouvant des idées inspirées notamment de Freire et Fals Borda: les connaissances doivent contribuer à une transformation de la réalité individuelle et collective, mettre à jour les inégalités sociales ainsi que favoriser l'empowerment et l'émancipation (Anadón 2006 ; Hall, Edward et Tandon 2016). La posture épistémologique féministe du « point de vue situé " (Collins 1990) en est un exemple: développer des savoirs grâce à une coopération entre des personnes directement interpellées, conscientes et engagées qui mobilisent leurs propres capacités pour se changer elles-mêmes ainsi que leurs réalités. Ces dernières produisent des connaissances utiles pour elles-mêmes et leurs conditions d'existence, lesquelles s'appuient sur une réflexivité critique à l'égard, notamment, des dynamiques de production du savoir qui reproduisent les privilèges, les oppressions, et discréditent leurs paroles, expériences et savoirs. 
Présente dans l'ensemble des disciplines, en anthropologie et plus largement en sciences sociales, la recherche participative est liée assez étroitement à la recherche qualitative avec laquelle elle partage plusieurs éléments. Elle est en rupture avec la tradition positiviste et remet fortement en question des critères tels que l'objectivité, l'autorité, la neutralité. Cette stratégie permet de rendre compte non seulement d'une réalité complexe dans laquelle il y a contingence et imprévisibilité, mais aussi de la nature multi-référentielle de cette réalité. Cette dernière peut être appréhendée selon plusieurs systèmes interprétatifs, donner lieu à une pluralité de discours rationnels et ceux-ci, en complémentarité, peuvent l'éclairer de façon heuristique. La recherche participative est donc enracinée dans un terrain et elle se fait résolument interprétative - plusieurs parlent, à la suite de Glaser et Strauss (1967), de grounded theory. Elle reconnaît également les savoirs expérientiels et élabore des processus de recherche qui co-construisent des connaissances de concert avec les acteurs et actrices concerné.e.s et bien souvent dans l'interdisciplinarité. Elle comprend l'objet de la recherche à partir de la perspective de la (ou des) communauté(s), analyse la signification que les gens lui donnent et explore avec eux les solutions appropriées. Cela exige que les partenaires partagent des valeurs et des intérêts connexes, et développent une relation de confiance qui s'accroît souvent quand les liens s'inscrivent dans le temps (Audoux et Gillet, 2011).

Dans un refus de la scission classique entre " expert " et " néophyte ", " chercheur » et " praticien » il s'agit de croiser différentes communautés de savoirs qui détiennent chacune ses propres sources et modes d'élaboration de la connaissance. Les recherches menées témoignent d'une préoccupation pour l'égalité des partenaires ou, du moins, pour un principe d'équité. Cependant, le plus souvent, il y a reconnaissance des différences, et les processus mis en place n'exigent pas que toutes et tous jouent le même rôle. II n'est pas question ici de fusion : les gens conservent leur identité première. C'est de la rencontre entre divers mondes que provient l'originalité des connaissances développées et l'innovation (Bonny 2017 : 45). Cette rencontre implique une forte réflexivité de la part de toutes et tous, «[...] réflexivité [qui] permet de capter une pratique en train de se faire et de se dire, un "savoir" en train de se construire " (Desgagné et al. 2001 : 38). Elle exige également une aptitude à discuter (voire à débattre) et à accepter un décentrement (voire une remise en cause) des cadres de références de chacun.e. S'invitent ainsi, dans les critères de scientificité, des critères d'ordre éthique fondamentaux, comme le respect du point de vue de l'autre, les modalités de son inclusion dans le processus de recherche, les impacts individuels et collectifs de l'investigation.

Au cœur donc de la recherche participative se retrouve cette idée d'une coconstruction des savoirs, et ce, peu importe la méthodologie adoptée qui peut être extrêmement variée: notes de terrain ethnologique, entrevues, observations (participantes ou non), récits de vie, inventaires, créations littéraires, œuvres participatives, etc. Cet espace réflexif créé prend souvent, a minima, la forme d'un comité d'élaboration/encadrement qui regroupe les acteurs et actrices mobilisé.e.s des milieux scientifiques et sociaux. La recherche peut également être encadrée par une instance de médiation, comme le fait le Service aux collectivités (SAC) actif depuis plus de trente ans à l'Université du Québec à Montréal (UQAM). Celui-ci soutient, entre autres, les recherches partenariales université/communauté en leur associant une 
personne qui veille, grâce à son expertise, au bon déroulement des choses, au respect des exigences de chacun.e et de son milieu respectif, à la résolution de conflits et à l'élaboration d'outils de protection juridique (contrat entre partenaires, entente sur la propriété intellectuelle et les modalités de diffusion, etc.).

Les niveaux de participation rencontrés sont très variables, passant d'un statut de collaborateur/collaboratrice, à partenaire, voire à cochercheur.e - ce dernier statut étant présent surtout dans la recherche-action. Desgagné (2007: 89-121) identifie trois niveaux: "cosituer la recherche » (définir l'objet et le cadre théorique), " coopérer la recherche" (définir la méthodologie et les stratégies de collecte de données) et " coproduire la recherche " (effectuer la collecte de données, l'analyse de celles-ci et la présentation des résultats). La majorité des auteur.e.s s'entendent pour dire que le "co » exige minimalement l'identification d'un objet de recherche partagé, de même qu'une prise en compte, à chacune des étapes, des préoccupations de l'ensemble des partenaires et du monde qu'elles/ils représentent. En outre, les acteurs et actrices issu.e.s du terrain sont très souvent fortement engagé.e.s dans la validation et la bonification de l'analyse des données, de même que dans la diffusion des résultats. Cette dernière étape est fondamentale dans la recherche participative, et souvent exigeante, du moins pour les chercheur.e.s qui doivent une fois de plus sortir de leur zone de confort, créer avec d'autres des formats de diffusion ou de transfert de connaissances innovants qui pourront servir concrètement aux milieux et être facilement appropriables par leurs acteurs et actrices, et enfin s'assurer d'une diffusion dans le milieu académique - sous les formes plus classiques de rapports de recherche, d'articles, de livres, de conférences, etc.

On affirme souvent que la recherche participative est une recherche "pour » et "avec » et non "sur». Avec Sebiotte (2007: 71-71), cependant, nous pensons qu'elle recoupe ces trois termes, mais selon des acceptions spécifiques: "pour" traduit la finalité des connaissances destinées aux praticiens et chercheurs ; "sur" traduit que les connaissances concernent des pratiques, des savoirs liés à l'action dans des milieux complexes ; "avec" exprime que c'est le seul moyen d'y arriver ». Et la/le chercheur.e détient un rôle central. Comme personne organisatrice, elle doit mettre en place le dispositif de co-construction de la recherche et le réguler. C'est elle qui est responsable de la qualité de la collaboration et de sa dimension éthique, de même que de s'assurer de la rigueur scientifique déployée. Comme partenaire, elle ou il doit mobiliser son expertise, non pas dans l'objectif de critiquer la pratique, mais bien plutôt de contribuer à mieux la saisir, la contextualiser, de même que, du moins idéalement, concourir à son évolution.

\section{Références}

Anadón, M. (2006), « La recherche dite "qualitative" : de la dynamique de son évolution aux acquis indéniables et aux questionnements présents ». Recherches qualitatives, vol.26, n¹, p.5-31. 
Audoux, C. et A. Gillet (2011), « Recherche partenariale et co-construction de savoirs entre chercheurs et acteurs : l'épreuve de la traduction ». Interventions économiques, $\mathrm{n}^{\circ} 43$. https://journals.openedition.org/interventionseconomiques/1347 https://doi.org/10.4000/interventionseconomiques.1347

Bonny, Y., (2017), « Les recherches partenariales participatives : éléments d'analyse et de typologie ». Dans A. Gillet et D.-G. Tremblay (dir.), Les recherches partenariales et collaboratives, Québec, Presses de l'Université du Québec, p.25-44. https://doi.org/10.2307/j.ctt1vw0rpg.3

Collins, P.H. (1990), Black Feminist Thought: Knowledge, Consciousness, and the Politics of Empowerment. New York, Routledge.

Desgagné, S., N. Bednarz, C. Couture, L. Poirier et P. Lebuis (2001), « L'approche collaborative de recherche en éducation : un rapport nouveau à établir entre recherche et formation ». Revue des sciences de l'éducation, vol.XXVII, n¹, p.33-64. https://doi.org/10.7202/000305ar

Desgagné, S. (2007), "Le défi de coproduction de "savoir" en recherche collaborative. Autour d'une démarche de reconstruction et d'analyse de récits de pratique enseignante ». Dans M. Anadón (dir.), La recherche participative. Multiples regards, Québec, Presses de l'Université Laval, p.89-121. https://doi.org/10.2307/j.ctv18ph9tc.7

Dewey, J. (1927), The Public and its Problem. New York, Henry Holt and Company.

Glaser, B.G. et A. Strauss (1967), The discovery of grounded theory: Strategies for qualitative research. Chicago, Aldine. https://doi.org/10.1097/00006199-196807000-00014 Hall, B., J. Edward et R. Tandon (2016), Knowledge, Democracy and Action: Community-University Research Partnerships in Global Perspectives. Oxford, Oxford University Press. https://doi.org/10.7765/9781526111418.00017

Sebiotte, M. (2007), « L'analyse des pratiques. Réflexions épistémologiques pour l'agir du chercheur ». Dans M. Anadón (dir.), La recherche participative. Multiples regards, Québec, Presses de l’Université Laval, p.49-87. https://doi.org/10.2307/j.ctv18ph9tc.6 